\title{
A Syntactic Approach to Unification in Transitive Reflexive Modal Logics
}

\author{
Rosalie lemhoff
}

\begin{abstract}
This paper contains a proof-theoretic account of unification in transitive reflexive modal logics, which means that the reasoning is syntactic and uses as little semantics as possible. New proofs of theorems on unification types are presented and these results are extended to negationless fragments. In particular, a syntactic proof of Ghilardi's result that S4 has finitary unification is provided. In this approach the relation between classical valuations, projective unifiers, and admissible rules is clarified.
\end{abstract}

\section{Introduction}

When restricted to propositional logic, unification theory is concerned with the problem whether a given formula can become derivable under a substitution. In general, a unification problem asks for the unifier of a pair of terms, or collection of pairs of terms, which in the context of a logic is a substitution under which two formulas become equivalent in the logic. This, however, can be reformulated as the problem of finding a substitution under which a formula becomes derivable. Such substitutions are called the unifiers of a formula.

In classical propositional logic every consistent formula has a unifier, because every satisfying valuation corresponds to a ground unifier that replaces the atoms in the formula by $\top$ or $\perp$. A substitution is a maximal unifier (mu) of a formula if among the unifiers of the formula it is maximal in the following ordering:

$$
\tau \leq \sigma \equiv_{\text {def }} \exists \pi(\tau=\mathrm{L} \pi \sigma),
$$

and it is a most general unifier $(m g u)$ if it is also unique modulo $=$, which is the intersection of $\leq$ and $\geq$. Here $={ }_{\mathrm{L}}$ is the equivalence relation on substitutions associated with the logic: $\sigma=\mathrm{L} \tau$ if and only if $\sigma(p) \leftrightarrow \tau(p)$ is derivable for all atoms $p$. If $\tau \leq \sigma$, we say that $\tau$ is less general than $\sigma$.

Received February 27, 2012; accepted December 30, 2013

First published online February 9, 2016

2010 Mathematics Subject Classification: Primary 03F03, 03B45; Secondary 03B70

Keywords: unification, admissible rules, modal logic, fragments

(C) 2016 by University of Notre Dame 10.1215/00294527-345997 


\title{
A Syntactic Approach to Unification in Transitive Reflexive Modal Logics
}

\author{
Rosalie lemhoff
}

\begin{abstract}
This paper contains a proof-theoretic account of unification in transitive reflexive modal logics, which means that the reasoning is syntactic and uses as little semantics as possible. New proofs of theorems on unification types are presented and these results are extended to negationless fragments. In particular, a syntactic proof of Ghilardi's result that S4 has finitary unification is provided. In this approach the relation between classical valuations, projective unifiers, and admissible rules is clarified.
\end{abstract}

\section{Introduction}

When restricted to propositional logic, unification theory is concerned with the problem whether a given formula can become derivable under a substitution. In general, a unification problem asks for the unifier of a pair of terms, or collection of pairs of terms, which in the context of a logic is a substitution under which two formulas become equivalent in the logic. This, however, can be reformulated as the problem of finding a substitution under which a formula becomes derivable. Such substitutions are called the unifiers of a formula.

In classical propositional logic every consistent formula has a unifier, because every satisfying valuation corresponds to a ground unifier that replaces the atoms in the formula by $\top$ or $\perp$. A substitution is a maximal unifier (mu) of a formula if among the unifiers of the formula it is maximal in the following ordering:

$$
\tau \leq \sigma \equiv_{\text {def }} \exists \pi(\tau=\mathrm{L} \pi \sigma),
$$

and it is a most general unifier $(m g u)$ if it is also unique modulo $=$, which is the intersection of $\leq$ and $\geq$. Here $={ }_{\mathrm{L}}$ is the equivalence relation on substitutions associated with the logic: $\sigma=\mathrm{L} \tau$ if and only if $\sigma(p) \leftrightarrow \tau(p)$ is derivable for all atoms $p$. If $\tau \leq \sigma$, we say that $\tau$ is less general than $\sigma$.

Received February 27, 2012; accepted December 30, 2013

First published online February 9, 2016

2010 Mathematics Subject Classification: Primary 03F03, 03B45; Secondary 03B70

Keywords: unification, admissible rules, modal logic, fragments

(C) 2016 by University of Notre Dame 10.1215/00294527-345997 


\title{
A Syntactic Approach to Unification in Transitive Reflexive Modal Logics
}

\author{
Rosalie lemhoff
}

\begin{abstract}
This paper contains a proof-theoretic account of unification in transitive reflexive modal logics, which means that the reasoning is syntactic and uses as little semantics as possible. New proofs of theorems on unification types are presented and these results are extended to negationless fragments. In particular, a syntactic proof of Ghilardi's result that S4 has finitary unification is provided. In this approach the relation between classical valuations, projective unifiers, and admissible rules is clarified.
\end{abstract}

\section{Introduction}

When restricted to propositional logic, unification theory is concerned with the problem whether a given formula can become derivable under a substitution. In general, a unification problem asks for the unifier of a pair of terms, or collection of pairs of terms, which in the context of a logic is a substitution under which two formulas become equivalent in the logic. This, however, can be reformulated as the problem of finding a substitution under which a formula becomes derivable. Such substitutions are called the unifiers of a formula.

In classical propositional logic every consistent formula has a unifier, because every satisfying valuation corresponds to a ground unifier that replaces the atoms in the formula by $\top$ or $\perp$. A substitution is a maximal unifier (mu) of a formula if among the unifiers of the formula it is maximal in the following ordering:

$$
\tau \leq \sigma \equiv_{\text {def }} \exists \pi(\tau=\mathrm{L} \pi \sigma),
$$

and it is a most general unifier $(m g u)$ if it is also unique modulo $=$, which is the intersection of $\leq$ and $\geq$. Here $={ }_{\mathrm{L}}$ is the equivalence relation on substitutions associated with the logic: $\sigma=\mathrm{L} \tau$ if and only if $\sigma(p) \leftrightarrow \tau(p)$ is derivable for all atoms $p$. If $\tau \leq \sigma$, we say that $\tau$ is less general than $\sigma$.

Received February 27, 2012; accepted December 30, 2013

First published online February 9, 2016

2010 Mathematics Subject Classification: Primary 03F03, 03B45; Secondary 03B70

Keywords: unification, admissible rules, modal logic, fragments

(C) 2016 by University of Notre Dame 10.1215/00294527-345997 


\title{
A Syntactic Approach to Unification in Transitive Reflexive Modal Logics
}

\author{
Rosalie lemhoff
}

\begin{abstract}
This paper contains a proof-theoretic account of unification in transitive reflexive modal logics, which means that the reasoning is syntactic and uses as little semantics as possible. New proofs of theorems on unification types are presented and these results are extended to negationless fragments. In particular, a syntactic proof of Ghilardi's result that S4 has finitary unification is provided. In this approach the relation between classical valuations, projective unifiers, and admissible rules is clarified.
\end{abstract}

\section{Introduction}

When restricted to propositional logic, unification theory is concerned with the problem whether a given formula can become derivable under a substitution. In general, a unification problem asks for the unifier of a pair of terms, or collection of pairs of terms, which in the context of a logic is a substitution under which two formulas become equivalent in the logic. This, however, can be reformulated as the problem of finding a substitution under which a formula becomes derivable. Such substitutions are called the unifiers of a formula.

In classical propositional logic every consistent formula has a unifier, because every satisfying valuation corresponds to a ground unifier that replaces the atoms in the formula by $\top$ or $\perp$. A substitution is a maximal unifier (mu) of a formula if among the unifiers of the formula it is maximal in the following ordering:

$$
\tau \leq \sigma \equiv_{\text {def }} \exists \pi(\tau=\mathrm{L} \pi \sigma),
$$

and it is a most general unifier $(m g u)$ if it is also unique modulo $=$, which is the intersection of $\leq$ and $\geq$. Here $={ }_{\mathrm{L}}$ is the equivalence relation on substitutions associated with the logic: $\sigma=\mathrm{L} \tau$ if and only if $\sigma(p) \leftrightarrow \tau(p)$ is derivable for all atoms $p$. If $\tau \leq \sigma$, we say that $\tau$ is less general than $\sigma$.

Received February 27, 2012; accepted December 30, 2013

First published online February 9, 2016

2010 Mathematics Subject Classification: Primary 03F03, 03B45; Secondary 03B70

Keywords: unification, admissible rules, modal logic, fragments

(C) 2016 by University of Notre Dame 10.1215/00294527-345997 


\title{
A Syntactic Approach to Unification in Transitive Reflexive Modal Logics
}

\author{
Rosalie lemhoff
}

\begin{abstract}
This paper contains a proof-theoretic account of unification in transitive reflexive modal logics, which means that the reasoning is syntactic and uses as little semantics as possible. New proofs of theorems on unification types are presented and these results are extended to negationless fragments. In particular, a syntactic proof of Ghilardi's result that S4 has finitary unification is provided. In this approach the relation between classical valuations, projective unifiers, and admissible rules is clarified.
\end{abstract}

\section{Introduction}

When restricted to propositional logic, unification theory is concerned with the problem whether a given formula can become derivable under a substitution. In general, a unification problem asks for the unifier of a pair of terms, or collection of pairs of terms, which in the context of a logic is a substitution under which two formulas become equivalent in the logic. This, however, can be reformulated as the problem of finding a substitution under which a formula becomes derivable. Such substitutions are called the unifiers of a formula.

In classical propositional logic every consistent formula has a unifier, because every satisfying valuation corresponds to a ground unifier that replaces the atoms in the formula by $\top$ or $\perp$. A substitution is a maximal unifier (mu) of a formula if among the unifiers of the formula it is maximal in the following ordering:

$$
\tau \leq \sigma \equiv_{\text {def }} \exists \pi(\tau=\mathrm{L} \pi \sigma),
$$

and it is a most general unifier $(m g u)$ if it is also unique modulo $=$, which is the intersection of $\leq$ and $\geq$. Here $={ }_{\mathrm{L}}$ is the equivalence relation on substitutions associated with the logic: $\sigma=\mathrm{L} \tau$ if and only if $\sigma(p) \leftrightarrow \tau(p)$ is derivable for all atoms $p$. If $\tau \leq \sigma$, we say that $\tau$ is less general than $\sigma$.

Received February 27, 2012; accepted December 30, 2013

First published online February 9, 2016

2010 Mathematics Subject Classification: Primary 03F03, 03B45; Secondary 03B70

Keywords: unification, admissible rules, modal logic, fragments

(C) 2016 by University of Notre Dame 10.1215/00294527-345997 


\title{
A Syntactic Approach to Unification in Transitive Reflexive Modal Logics
}

\author{
Rosalie lemhoff
}

\begin{abstract}
This paper contains a proof-theoretic account of unification in transitive reflexive modal logics, which means that the reasoning is syntactic and uses as little semantics as possible. New proofs of theorems on unification types are presented and these results are extended to negationless fragments. In particular, a syntactic proof of Ghilardi's result that S4 has finitary unification is provided. In this approach the relation between classical valuations, projective unifiers, and admissible rules is clarified.
\end{abstract}

\section{Introduction}

When restricted to propositional logic, unification theory is concerned with the problem whether a given formula can become derivable under a substitution. In general, a unification problem asks for the unifier of a pair of terms, or collection of pairs of terms, which in the context of a logic is a substitution under which two formulas become equivalent in the logic. This, however, can be reformulated as the problem of finding a substitution under which a formula becomes derivable. Such substitutions are called the unifiers of a formula.

In classical propositional logic every consistent formula has a unifier, because every satisfying valuation corresponds to a ground unifier that replaces the atoms in the formula by $\top$ or $\perp$. A substitution is a maximal unifier (mu) of a formula if among the unifiers of the formula it is maximal in the following ordering:

$$
\tau \leq \sigma \equiv_{\text {def }} \exists \pi(\tau=\mathrm{L} \pi \sigma),
$$

and it is a most general unifier $(m g u)$ if it is also unique modulo $=$, which is the intersection of $\leq$ and $\geq$. Here $={ }_{\mathrm{L}}$ is the equivalence relation on substitutions associated with the logic: $\sigma=\mathrm{L} \tau$ if and only if $\sigma(p) \leftrightarrow \tau(p)$ is derivable for all atoms $p$. If $\tau \leq \sigma$, we say that $\tau$ is less general than $\sigma$.

Received February 27, 2012; accepted December 30, 2013

First published online February 9, 2016

2010 Mathematics Subject Classification: Primary 03F03, 03B45; Secondary 03B70

Keywords: unification, admissible rules, modal logic, fragments

(C) 2016 by University of Notre Dame 10.1215/00294527-345997 


\title{
A Syntactic Approach to Unification in Transitive Reflexive Modal Logics
}

\author{
Rosalie lemhoff
}

\begin{abstract}
This paper contains a proof-theoretic account of unification in transitive reflexive modal logics, which means that the reasoning is syntactic and uses as little semantics as possible. New proofs of theorems on unification types are presented and these results are extended to negationless fragments. In particular, a syntactic proof of Ghilardi's result that S4 has finitary unification is provided. In this approach the relation between classical valuations, projective unifiers, and admissible rules is clarified.
\end{abstract}

\section{Introduction}

When restricted to propositional logic, unification theory is concerned with the problem whether a given formula can become derivable under a substitution. In general, a unification problem asks for the unifier of a pair of terms, or collection of pairs of terms, which in the context of a logic is a substitution under which two formulas become equivalent in the logic. This, however, can be reformulated as the problem of finding a substitution under which a formula becomes derivable. Such substitutions are called the unifiers of a formula.

In classical propositional logic every consistent formula has a unifier, because every satisfying valuation corresponds to a ground unifier that replaces the atoms in the formula by $\top$ or $\perp$. A substitution is a maximal unifier (mu) of a formula if among the unifiers of the formula it is maximal in the following ordering:

$$
\tau \leq \sigma \equiv_{\text {def }} \exists \pi(\tau=\mathrm{L} \pi \sigma),
$$

and it is a most general unifier $(m g u)$ if it is also unique modulo $=$, which is the intersection of $\leq$ and $\geq$. Here $={ }_{\mathrm{L}}$ is the equivalence relation on substitutions associated with the logic: $\sigma=\mathrm{L} \tau$ if and only if $\sigma(p) \leftrightarrow \tau(p)$ is derivable for all atoms $p$. If $\tau \leq \sigma$, we say that $\tau$ is less general than $\sigma$.

Received February 27, 2012; accepted December 30, 2013

First published online February 9, 2016

2010 Mathematics Subject Classification: Primary 03F03, 03B45; Secondary 03B70

Keywords: unification, admissible rules, modal logic, fragments

(C) 2016 by University of Notre Dame 10.1215/00294527-345997 


\title{
A Syntactic Approach to Unification in Transitive Reflexive Modal Logics
}

\author{
Rosalie lemhoff
}

\begin{abstract}
This paper contains a proof-theoretic account of unification in transitive reflexive modal logics, which means that the reasoning is syntactic and uses as little semantics as possible. New proofs of theorems on unification types are presented and these results are extended to negationless fragments. In particular, a syntactic proof of Ghilardi's result that S4 has finitary unification is provided. In this approach the relation between classical valuations, projective unifiers, and admissible rules is clarified.
\end{abstract}

\section{Introduction}

When restricted to propositional logic, unification theory is concerned with the problem whether a given formula can become derivable under a substitution. In general, a unification problem asks for the unifier of a pair of terms, or collection of pairs of terms, which in the context of a logic is a substitution under which two formulas become equivalent in the logic. This, however, can be reformulated as the problem of finding a substitution under which a formula becomes derivable. Such substitutions are called the unifiers of a formula.

In classical propositional logic every consistent formula has a unifier, because every satisfying valuation corresponds to a ground unifier that replaces the atoms in the formula by $\top$ or $\perp$. A substitution is a maximal unifier (mu) of a formula if among the unifiers of the formula it is maximal in the following ordering:

$$
\tau \leq \sigma \equiv_{\text {def }} \exists \pi(\tau=\mathrm{L} \pi \sigma),
$$

and it is a most general unifier $(m g u)$ if it is also unique modulo $=$, which is the intersection of $\leq$ and $\geq$. Here $={ }_{\mathrm{L}}$ is the equivalence relation on substitutions associated with the logic: $\sigma=\mathrm{L} \tau$ if and only if $\sigma(p) \leftrightarrow \tau(p)$ is derivable for all atoms $p$. If $\tau \leq \sigma$, we say that $\tau$ is less general than $\sigma$.

Received February 27, 2012; accepted December 30, 2013

First published online February 9, 2016

2010 Mathematics Subject Classification: Primary 03F03, 03B45; Secondary 03B70

Keywords: unification, admissible rules, modal logic, fragments

(C) 2016 by University of Notre Dame 10.1215/00294527-345997 


\title{
A Syntactic Approach to Unification in Transitive Reflexive Modal Logics
}

\author{
Rosalie lemhoff
}

\begin{abstract}
This paper contains a proof-theoretic account of unification in transitive reflexive modal logics, which means that the reasoning is syntactic and uses as little semantics as possible. New proofs of theorems on unification types are presented and these results are extended to negationless fragments. In particular, a syntactic proof of Ghilardi's result that S4 has finitary unification is provided. In this approach the relation between classical valuations, projective unifiers, and admissible rules is clarified.
\end{abstract}

\section{Introduction}

When restricted to propositional logic, unification theory is concerned with the problem whether a given formula can become derivable under a substitution. In general, a unification problem asks for the unifier of a pair of terms, or collection of pairs of terms, which in the context of a logic is a substitution under which two formulas become equivalent in the logic. This, however, can be reformulated as the problem of finding a substitution under which a formula becomes derivable. Such substitutions are called the unifiers of a formula.

In classical propositional logic every consistent formula has a unifier, because every satisfying valuation corresponds to a ground unifier that replaces the atoms in the formula by $\top$ or $\perp$. A substitution is a maximal unifier (mu) of a formula if among the unifiers of the formula it is maximal in the following ordering:

$$
\tau \leq \sigma \equiv_{\text {def }} \exists \pi(\tau=\mathrm{L} \pi \sigma),
$$

and it is a most general unifier $(m g u)$ if it is also unique modulo $=$, which is the intersection of $\leq$ and $\geq$. Here $={ }_{\mathrm{L}}$ is the equivalence relation on substitutions associated with the logic: $\sigma=\mathrm{L} \tau$ if and only if $\sigma(p) \leftrightarrow \tau(p)$ is derivable for all atoms $p$. If $\tau \leq \sigma$, we say that $\tau$ is less general than $\sigma$.

Received February 27, 2012; accepted December 30, 2013

First published online February 9, 2016

2010 Mathematics Subject Classification: Primary 03F03, 03B45; Secondary 03B70

Keywords: unification, admissible rules, modal logic, fragments

(C) 2016 by University of Notre Dame 10.1215/00294527-345997 


\title{
A Syntactic Approach to Unification in Transitive Reflexive Modal Logics
}

\author{
Rosalie lemhoff
}

\begin{abstract}
This paper contains a proof-theoretic account of unification in transitive reflexive modal logics, which means that the reasoning is syntactic and uses as little semantics as possible. New proofs of theorems on unification types are presented and these results are extended to negationless fragments. In particular, a syntactic proof of Ghilardi's result that S4 has finitary unification is provided. In this approach the relation between classical valuations, projective unifiers, and admissible rules is clarified.
\end{abstract}

\section{Introduction}

When restricted to propositional logic, unification theory is concerned with the problem whether a given formula can become derivable under a substitution. In general, a unification problem asks for the unifier of a pair of terms, or collection of pairs of terms, which in the context of a logic is a substitution under which two formulas become equivalent in the logic. This, however, can be reformulated as the problem of finding a substitution under which a formula becomes derivable. Such substitutions are called the unifiers of a formula.

In classical propositional logic every consistent formula has a unifier, because every satisfying valuation corresponds to a ground unifier that replaces the atoms in the formula by $\top$ or $\perp$. A substitution is a maximal unifier (mu) of a formula if among the unifiers of the formula it is maximal in the following ordering:

$$
\tau \leq \sigma \equiv_{\text {def }} \exists \pi(\tau=\mathrm{L} \pi \sigma),
$$

and it is a most general unifier $(m g u)$ if it is also unique modulo $=$, which is the intersection of $\leq$ and $\geq$. Here $={ }_{\mathrm{L}}$ is the equivalence relation on substitutions associated with the logic: $\sigma=\mathrm{L} \tau$ if and only if $\sigma(p) \leftrightarrow \tau(p)$ is derivable for all atoms $p$. If $\tau \leq \sigma$, we say that $\tau$ is less general than $\sigma$.

Received February 27, 2012; accepted December 30, 2013

First published online February 9, 2016

2010 Mathematics Subject Classification: Primary 03F03, 03B45; Secondary 03B70

Keywords: unification, admissible rules, modal logic, fragments

(C) 2016 by University of Notre Dame 10.1215/00294527-345997 


\title{
A Syntactic Approach to Unification in Transitive Reflexive Modal Logics
}

\author{
Rosalie lemhoff
}

\begin{abstract}
This paper contains a proof-theoretic account of unification in transitive reflexive modal logics, which means that the reasoning is syntactic and uses as little semantics as possible. New proofs of theorems on unification types are presented and these results are extended to negationless fragments. In particular, a syntactic proof of Ghilardi's result that S4 has finitary unification is provided. In this approach the relation between classical valuations, projective unifiers, and admissible rules is clarified.
\end{abstract}

\section{Introduction}

When restricted to propositional logic, unification theory is concerned with the problem whether a given formula can become derivable under a substitution. In general, a unification problem asks for the unifier of a pair of terms, or collection of pairs of terms, which in the context of a logic is a substitution under which two formulas become equivalent in the logic. This, however, can be reformulated as the problem of finding a substitution under which a formula becomes derivable. Such substitutions are called the unifiers of a formula.

In classical propositional logic every consistent formula has a unifier, because every satisfying valuation corresponds to a ground unifier that replaces the atoms in the formula by $\top$ or $\perp$. A substitution is a maximal unifier (mu) of a formula if among the unifiers of the formula it is maximal in the following ordering:

$$
\tau \leq \sigma \equiv_{\text {def }} \exists \pi(\tau=\mathrm{L} \pi \sigma),
$$

and it is a most general unifier $(m g u)$ if it is also unique modulo $=$, which is the intersection of $\leq$ and $\geq$. Here $={ }_{\mathrm{L}}$ is the equivalence relation on substitutions associated with the logic: $\sigma=\mathrm{L} \tau$ if and only if $\sigma(p) \leftrightarrow \tau(p)$ is derivable for all atoms $p$. If $\tau \leq \sigma$, we say that $\tau$ is less general than $\sigma$.

Received February 27, 2012; accepted December 30, 2013

First published online February 9, 2016

2010 Mathematics Subject Classification: Primary 03F03, 03B45; Secondary 03B70

Keywords: unification, admissible rules, modal logic, fragments

(C) 2016 by University of Notre Dame 10.1215/00294527-345997 


\title{
A Syntactic Approach to Unification in Transitive Reflexive Modal Logics
}

\author{
Rosalie lemhoff
}

\begin{abstract}
This paper contains a proof-theoretic account of unification in transitive reflexive modal logics, which means that the reasoning is syntactic and uses as little semantics as possible. New proofs of theorems on unification types are presented and these results are extended to negationless fragments. In particular, a syntactic proof of Ghilardi's result that S4 has finitary unification is provided. In this approach the relation between classical valuations, projective unifiers, and admissible rules is clarified.
\end{abstract}

\section{Introduction}

When restricted to propositional logic, unification theory is concerned with the problem whether a given formula can become derivable under a substitution. In general, a unification problem asks for the unifier of a pair of terms, or collection of pairs of terms, which in the context of a logic is a substitution under which two formulas become equivalent in the logic. This, however, can be reformulated as the problem of finding a substitution under which a formula becomes derivable. Such substitutions are called the unifiers of a formula.

In classical propositional logic every consistent formula has a unifier, because every satisfying valuation corresponds to a ground unifier that replaces the atoms in the formula by $\top$ or $\perp$. A substitution is a maximal unifier (mu) of a formula if among the unifiers of the formula it is maximal in the following ordering:

$$
\tau \leq \sigma \equiv_{\text {def }} \exists \pi(\tau=\mathrm{L} \pi \sigma),
$$

and it is a most general unifier $(m g u)$ if it is also unique modulo $=$, which is the intersection of $\leq$ and $\geq$. Here $={ }_{\mathrm{L}}$ is the equivalence relation on substitutions associated with the logic: $\sigma=\mathrm{L} \tau$ if and only if $\sigma(p) \leftrightarrow \tau(p)$ is derivable for all atoms $p$. If $\tau \leq \sigma$, we say that $\tau$ is less general than $\sigma$.

Received February 27, 2012; accepted December 30, 2013

First published online February 9, 2016

2010 Mathematics Subject Classification: Primary 03F03, 03B45; Secondary 03B70

Keywords: unification, admissible rules, modal logic, fragments

(C) 2016 by University of Notre Dame 10.1215/00294527-345997 


\title{
A Syntactic Approach to Unification in Transitive Reflexive Modal Logics
}

\author{
Rosalie lemhoff
}

\begin{abstract}
This paper contains a proof-theoretic account of unification in transitive reflexive modal logics, which means that the reasoning is syntactic and uses as little semantics as possible. New proofs of theorems on unification types are presented and these results are extended to negationless fragments. In particular, a syntactic proof of Ghilardi's result that S4 has finitary unification is provided. In this approach the relation between classical valuations, projective unifiers, and admissible rules is clarified.
\end{abstract}

\section{Introduction}

When restricted to propositional logic, unification theory is concerned with the problem whether a given formula can become derivable under a substitution. In general, a unification problem asks for the unifier of a pair of terms, or collection of pairs of terms, which in the context of a logic is a substitution under which two formulas become equivalent in the logic. This, however, can be reformulated as the problem of finding a substitution under which a formula becomes derivable. Such substitutions are called the unifiers of a formula.

In classical propositional logic every consistent formula has a unifier, because every satisfying valuation corresponds to a ground unifier that replaces the atoms in the formula by $\top$ or $\perp$. A substitution is a maximal unifier (mu) of a formula if among the unifiers of the formula it is maximal in the following ordering:

$$
\tau \leq \sigma \equiv_{\text {def }} \exists \pi(\tau=\mathrm{L} \pi \sigma),
$$

and it is a most general unifier $(m g u)$ if it is also unique modulo $=$, which is the intersection of $\leq$ and $\geq$. Here $={ }_{\mathrm{L}}$ is the equivalence relation on substitutions associated with the logic: $\sigma=\mathrm{L} \tau$ if and only if $\sigma(p) \leftrightarrow \tau(p)$ is derivable for all atoms $p$. If $\tau \leq \sigma$, we say that $\tau$ is less general than $\sigma$.

Received February 27, 2012; accepted December 30, 2013

First published online February 9, 2016

2010 Mathematics Subject Classification: Primary 03F03, 03B45; Secondary 03B70

Keywords: unification, admissible rules, modal logic, fragments

(C) 2016 by University of Notre Dame 10.1215/00294527-345997 


\title{
A Syntactic Approach to Unification in Transitive Reflexive Modal Logics
}

\author{
Rosalie lemhoff
}

\begin{abstract}
This paper contains a proof-theoretic account of unification in transitive reflexive modal logics, which means that the reasoning is syntactic and uses as little semantics as possible. New proofs of theorems on unification types are presented and these results are extended to negationless fragments. In particular, a syntactic proof of Ghilardi's result that S4 has finitary unification is provided. In this approach the relation between classical valuations, projective unifiers, and admissible rules is clarified.
\end{abstract}

\section{Introduction}

When restricted to propositional logic, unification theory is concerned with the problem whether a given formula can become derivable under a substitution. In general, a unification problem asks for the unifier of a pair of terms, or collection of pairs of terms, which in the context of a logic is a substitution under which two formulas become equivalent in the logic. This, however, can be reformulated as the problem of finding a substitution under which a formula becomes derivable. Such substitutions are called the unifiers of a formula.

In classical propositional logic every consistent formula has a unifier, because every satisfying valuation corresponds to a ground unifier that replaces the atoms in the formula by $\top$ or $\perp$. A substitution is a maximal unifier (mu) of a formula if among the unifiers of the formula it is maximal in the following ordering:

$$
\tau \leq \sigma \equiv_{\text {def }} \exists \pi(\tau=\mathrm{L} \pi \sigma),
$$

and it is a most general unifier $(m g u)$ if it is also unique modulo $=$, which is the intersection of $\leq$ and $\geq$. Here $={ }_{\mathrm{L}}$ is the equivalence relation on substitutions associated with the logic: $\sigma=\mathrm{L} \tau$ if and only if $\sigma(p) \leftrightarrow \tau(p)$ is derivable for all atoms $p$. If $\tau \leq \sigma$, we say that $\tau$ is less general than $\sigma$.

Received February 27, 2012; accepted December 30, 2013

First published online February 9, 2016

2010 Mathematics Subject Classification: Primary 03F03, 03B45; Secondary 03B70

Keywords: unification, admissible rules, modal logic, fragments

(C) 2016 by University of Notre Dame 10.1215/00294527-345997 


\title{
A Syntactic Approach to Unification in Transitive Reflexive Modal Logics
}

\author{
Rosalie lemhoff
}

\begin{abstract}
This paper contains a proof-theoretic account of unification in transitive reflexive modal logics, which means that the reasoning is syntactic and uses as little semantics as possible. New proofs of theorems on unification types are presented and these results are extended to negationless fragments. In particular, a syntactic proof of Ghilardi's result that S4 has finitary unification is provided. In this approach the relation between classical valuations, projective unifiers, and admissible rules is clarified.
\end{abstract}

\section{Introduction}

When restricted to propositional logic, unification theory is concerned with the problem whether a given formula can become derivable under a substitution. In general, a unification problem asks for the unifier of a pair of terms, or collection of pairs of terms, which in the context of a logic is a substitution under which two formulas become equivalent in the logic. This, however, can be reformulated as the problem of finding a substitution under which a formula becomes derivable. Such substitutions are called the unifiers of a formula.

In classical propositional logic every consistent formula has a unifier, because every satisfying valuation corresponds to a ground unifier that replaces the atoms in the formula by $\top$ or $\perp$. A substitution is a maximal unifier (mu) of a formula if among the unifiers of the formula it is maximal in the following ordering:

$$
\tau \leq \sigma \equiv_{\text {def }} \exists \pi(\tau=\mathrm{L} \pi \sigma),
$$

and it is a most general unifier $(m g u)$ if it is also unique modulo $=$, which is the intersection of $\leq$ and $\geq$. Here $={ }_{\mathrm{L}}$ is the equivalence relation on substitutions associated with the logic: $\sigma=\mathrm{L} \tau$ if and only if $\sigma(p) \leftrightarrow \tau(p)$ is derivable for all atoms $p$. If $\tau \leq \sigma$, we say that $\tau$ is less general than $\sigma$.

Received February 27, 2012; accepted December 30, 2013

First published online February 9, 2016

2010 Mathematics Subject Classification: Primary 03F03, 03B45; Secondary 03B70

Keywords: unification, admissible rules, modal logic, fragments

(C) 2016 by University of Notre Dame 10.1215/00294527-345997 